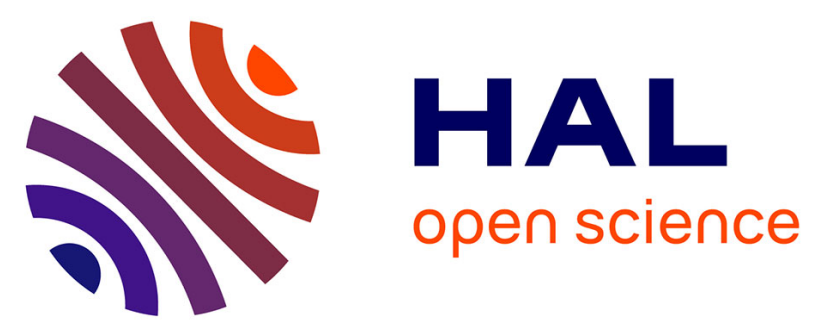

\title{
Resultados oncológicos y características patológicas de la sobrestadificación de cT1 a carcinoma de células renales pT3a en comparación con los tumores pT3a de novo
}

V. Senguttuvan Karthikeyan, A. Mallya, A. Sivaraman, M. Galiano, F. Rozet, N. Cathala, E. Barret, A. Mombet, D. Prapotnich, R. Sanchez-Salas, et al.

\section{To cite this version:}

V. Senguttuvan Karthikeyan, A. Mallya, A. Sivaraman, M. Galiano, F. Rozet, et al.. Resultados oncológicos y características patológicas de la sobrestadificación de cT1 a carcinoma de células renales pT3a en comparación con los tumores pT3a de novo. Actas Urológicas Españolas, 2019, 43, pp.234 240. 10.1016/j.acuro.2018.03.008 . hal-03484717

\section{HAL Id: hal-03484717 https://hal.science/hal-03484717}

Submitted on 21 Dec 2021

HAL is a multi-disciplinary open access archive for the deposit and dissemination of scientific research documents, whether they are published or not. The documents may come from teaching and research institutions in France or abroad, or from public or private research centers.
L'archive ouverte pluridisciplinaire HAL, est destinée au dépôt et à la diffusion de documents scientifiques de niveau recherche, publiés ou non, émanant des établissements d'enseignement et de recherche français ou étrangers, des laboratoires publics ou privés.

\section{(c) (1) $\$$}

Distributed under a Creative Commons Attribution - NonCommerciall 4.0 International 

carcinoma de células renales pT3a en comparación con los tumores de novo pT3a

6 Oncological outcomes and pathological characteristics of cT1 upstaging to pT3a renal cell carcinoma compared with denovo pT3a tumors

8

9 Running or Short title: "cT1 to pT3a upgraded RCC"

10 Título abreviado: "cT1 a CRR pT3a sobreestadificado"

1) Authors' names and Institutional affiliations:

1. V Senguttuvan Karthikeyan Department of Urology,

2. A Mallya

Department of Urology, Fortis Escorts Kidney and Urology Institute, New Delhi India.

3. A Sivaraman

Department of Urology, Institut Montsouris, Université Paris-Descartes. PARIS

4. M Galiano

Department of Urology, Institut Montsouris, Université Paris-Descartes. PARIS

\section{F Rozet}

Department of Urology, 
2

3

6. N Cathala

Department of Urology, Institut Montsouris, Université Paris-Descartes. PARIS

7. E Barret

Department of Urology, Institut Montsouris, Université Paris-Descartes. PARIS

8. A Mombet Department of Urology, Institut Montsouris, Université Paris-Descartes. PARIS

9. D Prapotnich Department of Urology, Institut Montsouris, Université Paris-Descartes. PARIS

10. R Sanchez-Salas**

Consultant Urologist, Department of Urology, Institut Montsouris, Université Paris-Descartes. PARIS

11. X Cathelineau

Department of Urology, Institut Montsouris, Université Paris-Descartes. PARIS

2) Corresponding author.

Dr. Rafael Sanchez-Salas

Email: raersas@gmail.com 
2 RESUMEN

3 Introducción

4 La importancia de la sobreestadificación de tumores renales cT1 a pT3a no está clara.

5 Evaluamos la incidencia de la sobreestadificación, identificamos factores predictivos y 6 analizamos los resultados oncológicos de estos pacientes frente a aquellos que no

7 sobreestadificaron. También comparamos los resultados oncológicos de la

8 sobreestadificación de cT1 a pT3a con tumores renales de novo pT3a.

9 Métodos

10 De una base de datos de 1021 tumores renales con datos de seguimiento completos 11 disponibles, 517 pacientes tenían cT1. Los pacientes que sobreestadificaron a pT3a se compararon con aquellos que no lo hicieron. Se analizaron los resultados de las características clínicas, perioperatorias, histopatológicas y oncológicas iniciales.

14 Resultados

De 517 pacientes con cT1, $105(20.3 \%)$ sobreestadificaron a pT3a y 412 (79.7\%) no lo hicieron. La proporción de pacientes en cada grupo sometidos a nefrectomía parcial y radical,

17 el tamaño del tumor postoperatorio, la histología, el estado de los márgenes, y la afectación de ganglios linfáticos fueron similares. Entre los que sobreestadificaron, $9(8,6 \%)$ pacientes desarrollaron la primera recurrencia en comparación con solo $3(0,7 \%)$ en aquellos que no sobreestadificaron $(\mathrm{p}<0,001)$. La mediana del tiempo hasta la recurrencia (57 frente a 107 meses; $\mathrm{p}<0,001)$ fue menor en los tumores renales denovo pT3a.

\section{Conclusiones} La sobreestadificación patológica de cT1 a pT3a y la necrosis en la histopatología se asociaron con la recurrencia. La edad avanzada, el tabaquismo, la necrosis en la histopatología, la histología de células claras y grados más altos de Fuhrman contribuyeron a la sobreestadificación patológica de los tumores cT1. El CCR de novo pT3a tuvo una 
1 supervivencia peor cuando se comparó con los pacientes con cT1 que sobreestadificaron a 2 CCR pT3a.

3 Palabras clave: tumores renales cT1, tumores renales pT3, sobreestadificación, recurrencia, 4 nefrectomía parcial

5

6

7 


\section{ABSTRACT}

5 Introduction

6 The significance of upstaging of cT1 renal tumors to pT3a is not clear. We evaluate the

7 incidence of upstaging, identify predictors and analyze oncological outcomes of these

8 patients versus those who did not upstage. We also compared the oncological outcomes of

9 cT1 upstaging to pT3a with denovo pT3a renal tumors.

10 Methods

11 From a database of 1021 renal tumors with complete available

12 follow-up data, 517 patients had cT1. Patients upstaging to pT3a were compared to those who 13 did not. Baseline clinical, perioperative, histopathologic features and oncological 14 outcomes were analysed.

15 Results

16 Out of 517 cT1 patients, $105(20.3 \%)$ upstaged to pT3a and $412(79.7 \%)$ did not. Proportion 17 of patients in each group undergoing partial and radical nephrectomy, postoperative tumor 18 size, histology, margin status and lymph node involvement were similar. Among upstaged, 9 (8.6\%) patients developed first recurrence as compared to only $3(0.7 \%)$ in those not upstaging $(\mathrm{p}<0.001)$.

21 The median time to recurrence (57 vs 107 months; $p<0.001$ ) was lesser in denovo pT3a 22 renal tumors.

23 Conclusions

24 Pathological upstaging from cT1 to pT3a and necrosis on histopathology were associated 25 with recurrence. Advanced age, smoking, necrosis on histopathology, clear cell histology and 
1 higher Fuhrman grades contributed to pathological upstaging of cT1 tumors. De novo pT3a

2 RCC had worse survival when compared to cT1 patients upstaging to pT3a RCC.

3

4 Palabras clave: cT1 tumor; pT3a tumor; upstage; recurrence; partial nephrectomy

\section{INTRODUCCIÓN}

Con la llegada de los avances en la toma de imágenes, la incidencia del carcinoma de células renales (CCR) va en aumento y más incidentalmente los CCR son clínicamente masas T1 (cT1) y tradicionalmente la nefrectomía radical (NR) ha sido el estándar de referencia para el manejo quirúrgico ${ }^{1}$. Sin embargo, con mejores resultados no oncológicos asociados con la preservación renal, hay un cambio de paradigma en el tratamiento hacia la nefrectomía parcial (NP) para CCR $\mathrm{cT}^{1,2}$. Posterior a esto, hay una sobreestadificación patológica, después de la escisión quirúrgica a T3a patológico (pT3a) ${ }^{1}$. El tamaño y el estadio tumoral son parámetros de pronóstico importantes en el CCR. Mientras que la sobreestadificación patológica de pT1 a pT2 se define por el tamaño, las características intrínsecas como la afectación de la vena renal o del seno renal pueden sobreestadificar cT1 a pT3a directamente, pero su importancia no es clara ${ }^{3,4}$

Los objetivos de este estudio fueron evaluar la incidencia de la sobreestadificación, identificar los factores predictivos y analizar los resultados oncológicos y las características 
1 patológicas con los pacientes que no sobreestadificaron. También comparamos los resultados

2 oncológicos y las características patológicas de la sobreestadificación de cT1 a pT3a con

3 tumores renales de novo pT3a.

4

5

\section{MÉTODOS}

7 Este fue un análisis de una base de datos de pacientes que pasaron de tumores renales de estadio cT1 a pT3a con diagnóstico de cáncer de células renales (CCR) en la histopatología final entre enero de 2007 y octubre de 2015 en el Departamento de Urología en un instituto de tercer nivel, en París, Francia. Se obtuvo la aprobacion de la junta de revisión institucional. De 517 cT1 CCRs con datos completos de seguimiento disponibles, 105 (20,3\%) pacientes pasaron a pT3a. Se excluyeron las lesiones que pasaron a pT3b y más allá. Se analizaron las características clínicas, perioperatorias, histopatológicas y los resultados oncológicos iniciales. También comparamos los resultados oncológicos de la sobreestadificación de cT1 a CCR de pT3a con 45 pacientes con CCR de novo pT3a durante el mismo período con datos completos de seguimiento disponibles.

\section{Evaluación}

La evaluación preoperatoria incluyó hemoglobina, creatinina sérica, electrolitos, ecografía y tomografía computarizada mejorada con contraste o toma de imágenes de la resonancia magnética del abdomen. Se realizaron creatinina sérica postoperatoria, electrolitos y hemoglobina. Se realizó histopatología postoperatoria y los pacientes fueron seguidos para la recurrencia. 


\section{$1 \quad$ Parámetros}

2 Se registraron los datos demográficos que incluyen edad, sexo, índice de masa corporal

3 (IMC), tabaquismo, tamaño tumoral y estadio preoperatorio y postoperatorio, ubicación y

4 clase ASA. Los detalles operativos incluyeron tipo de nefrectomía (NR o NP) y abordaje

5 (laparoscópico / abierto / robótico). Las complicaciones se clasificaron de acuerdo con el

6 sistema de clasificación modificado de Clavien y solo se analizó la complicación del grado

7 más alto. Se observaron histopatología postoperatoria, necrosis en EHP, grado de Fuhrman

8 y sobreestadificación a pT3a.

9

10 Procedimiento quirúrgico

11 Todas las nefrectomías fueron realizadas por un equipo de urólogos capacitados que tenían

12 al menos 5 años de experiencia en cirugías robóticas. Bajo anestesia general, se realizó NR o

13 NP abierta o laparoscópica o robótica. La nefrectomía robótica se realizó con el sistema

14 quirúrgico da Vinci (Intuitive Surgical, Sunnywale, California, EE. UU.). La laparoscopia y

15 los enfoques robóticos se adaptaron cuando los cirujanos se formaron en la técnica. 


\section{$2 \quad$ Análisis estadístico}

3 Los datos se analizaron utilizando SPSS v.20.0 (IBM Corp., Armonk, NY, EE. UU.). Las

4 variables continuas se representaron como media con desviación estándar y las variables

5 categóricas como mediana y rango intercuartil. La prueba $\mathrm{t}$ de Student (dos colas,

6 independiente) se usó para los parámetros métricos en escala continua entre dos grupos y la

7 prueba U de Mann Whitney se utilizó para variables discretas. Chi-cuadrado y la prueba

8 exacta de Fisher se utilizaron para comparar los parámetros en escala categórica entre dos o

9 más grupos. El análisis de regresión logística binomial se utilizó para identificar factores predictivos independientes de recidiva y sobreestadificación. Se realizó un análisis de supervivencia de Kaplan Meier para comparar la mediana de supervivencia entre la sobreestadificación de cT1 a pT3a con aquellos que no sobreestadificaron y el tiempo de recurrencia. También se realizó análisis de supervivencia para comparar la mediana de supervivencia entre cT1 que sobreestadificaron a pT3a con CCR de novo pT3a. Un valor p de $<0,05$ se consideró estadísticamente significativo.

\section{RESULTADOS}

\section{Características iniciales y operatorias}

Tuvimos 517 pacientes con tumores clínicos T1 en una base de datos de 1021 pacientes con tumores renales. Las características iniciales y operatorias se muestran en la Tabla 1. La mayoría $(433 ; 83,8 \%)$ de pacientes se sometió a cirugía laparoscópica, $56(10,8 \%)$ se sometieron a cirugía robótica y $28(5,4 \%)$ se sometieron a cirugía abierta. Los resultados de cT1 no se subclasificaron en cT1a y cT1b ya que no hubo diferencias estadísticamente significativas entre estos dos grupos. 
2 De 517 pacientes, $391(75.6 \%)$ no desarrollaron complicaciones que contribuyeran al grado

3 de Clavien 0. Las complicaciones de grado 1 de Clavien se observaron en $23(4,4 \%)$ 4 pacientes, Clavien 2 en $57(11,1 \%)$, Clavien 3 en $25(4,8 \%)$ y Clavien 4 en $9(1,8 \%)$ 5 pacientes. Doce $(2,3 \%)$ pacientes tuvieron muerte postoperatoria, que constituye Clavien 5 y $616(3,1 \%)$ pacientes necesitaron rehospitalización después del alta. El tamaño medio $( \pm \mathrm{DE})$ 7 del tumor postoperatorio fue de 39,56 \pm 17,53 mm. La histopatología reveló CCR de células claras (CCRCC) en $391(75,6 \%)$ pacientes, CCR papilar (CCRP) en $84(16,2 \%)$ y CCR cromófobo en $42(8,2 \%)$ pacientes, por lo tanto el no CCRCC contribuyó a $126(24,4 \%)$ pacientes. Se observó necrosis en $42(8,1 \%)$ pacientes y márgenes quirúrgicos positivos (MQP) en $11(2,1 \%)$ pacientes. Se observó Fuhrman grado 1 en $26(5 \%)$ grado 2 en 316 $(61,1 \%)$, grado 3 en $154(29,8 \%)$ y grado 4 en 21 (4,1\%) pacientes. En la histopatología final, se observaron tumores pT1a en $259(50,1 \%)$, pT1b en $142(27,5 \%)$, pT2a en $8(1,5 \%)$, pT2b en $3(0,6 \%)$ y pT3a en $105(20,3 \%)$ pacientes. Por lo tanto, de 517 pacientes, $105(20,3 \%)$ sobreestadificaron a pT3a y $412(79,7 \%)$ no lo hicieron. La creatinina sérica postoperatoria media $( \pm \mathrm{DE})$ en el último seguimiento fue de 110,27 $\pm 60,8 \mu \mathrm{mol} / \mathrm{L}$. Se observaron recurrencias en 15 (2,9\%) pacientes en un seguimiento mediano (RIQ) de 88 (204) meses con recurrencia en sitio distante en $7(46,7 \%)$ pacientes y recurrencia local en $14(93,3 \%)$ pacientes. Esto incluyó múltiples recurrencias. Doce $(2,3 \%)$ pacientes tuvieron la primera recurrencia. El período de tiempo mediano (RIQ) entre la primera y la segunda cirugía fue de 36 (93) meses. El EHP en la segunda cirugía fue CCRCC en 8, CCRP en 3 y benigno en 3 pacientes y 5 pacientes habían sobreestadificado a pT3a desde cT1.

\section{Recurrencia}

Los pacientes con recurrencia tenían características clínicas iniciales comparables como la 
1 edad, el sexo, el IMC, la clase ASA, el tamaño tumoral preoperatorio y postoperatorio, la

2 hemoglobina, la creatinina sérica, la ubicación del tumor con aquellos sin recurrencia. Una

3 proporción similar de pacientes en cada grupo se sometió a NR y NP (11: 4 en recurrencia

4 frente a 252: 250 en aquellos sin recurrencia, $\mathrm{p}=0,08)$. La sobreestadificación a pT3a, el

5 fumar y la necrosis en EHP fueron significativamente mayores $(p<0,001)$ en pacientes con

6 recurrencia. En la regresión logística binomial, el fumar $(\mathrm{p}<0,001)$, la presencia de necrosis

$7 \quad(\mathrm{p}<0,001)$ y sobreestadificación de pT3a $(\mathrm{p}<0,001)$ fueron factores predictivos significativos

8 de recurrencia.

\section{Sobreestadificación de pT3a}

11 La media de edad, el tabaquismo, el tamaño tumoral preoperatorio y postoperatorio, la necrosis en EHP y la histología de células claras fue significativamente mayor en los pacientes que sobreestadificaron (Tabla 2). Una clase ASA más alta y un grado más alto de Fuhrman se asociaron con la sobreestadificación de pT3a (Tabla 3). El estado de los márgenes quirúrgicos positivos y la afectación de ganglios linfáticos fueron similares. En la regresión logística binomial, el tamaño tumoral preoperatorio $(\mathrm{p}<0,001)$ y el estadio $\mathrm{T}$ postoperatorio $(\mathrm{p}=0,001)$ surgieron como factores predictivos significativos para sobreestadificación de pT3a.

Supervivencia

Cuando los pacientes sobreestadificaron a pT3a, la supervivencia fue significativamente menor (131; IC del 95\%-99,6 -162,39 meses) que aquellos que no sobreestadificaron (222; IC 95\% - 138,8 - 305,2 meses) en análisis de supervivencia (prueba de log rank; $p=0,015$ ) 
1 sobreestadificaron, $9(8,6 \%)$ pacientes desarrollaron la primera recurrencia en comparación

2 con solo $3(0,7 \%)$ en los que no sobreestadificaron ( $p<0,001)$. Sin embargo, la mediana del

3 tiempo hasta la recurrencia (152 meses frente a 139 meses, $p=0,76)$ fue similar (Figura 1B)

4 en ambos grupos.

5

$6 \quad$ Nefrectomía parcial

7 Entre las NP, el número de pacientes que sobreestadificaron fue de 27 (27/254, 10,6\%). En

8 la regresión logística binomial por sobreestadificación en pacientes con NP, el estadio

9 tumoral postoperatorio $(p<0,001)$, la clase ASA $(p<0,001)$ y la clase de complicaciones de

10 Clavien $(p=0,03)$ emergieron como factores predictivos significativos.

11

12

13

14

15

16

17

18

19

20

21

22

23

24 Tuvimos 45 pacientes con CCR de novo pT3a. Las características iniciales y operativas se

25 resumen en la Tabla 1. La histopatología reveló CCR de células claras (CCRCC) en 34

CCR de novo pT3a 
1 (75,6\%) pacientes, CCR papilar (CCRP) en $7(15,5 \%)$ y CCR cromófobo en $4(8,9 \%)$

2 pacientes. Se observó necrosis en $15(33,3 \%)$ pacientes y MQP en $3(6,7 \%)$ pacientes. Se

3 observó Fuhrman grado 1 en $3(6,7 \%)$, grado 2 en $6(13,3 \%)$, grado 3 en $26(57,8 \%)$ y grado

44 en $10(22,2 \%)$ pacientes. La media $( \pm \mathrm{DE})$ de creatinina sérica postoperatoria en el último

5 seguimiento fue de 117,56 $\pm 40,3 \mu \mathrm{mol} / \mathrm{L}$. Se observó recurrencia del sitio distante en 13

$6(28,9 \%)$ pacientes en un seguimiento mediano (RIQ) de 77 (183) meses. La mediana de

7 supervivencia (RIQ) en pacientes con cT1 que sobreestadifican a pT3a fue mejor que el CCR

8 de novo pT3a (107 frente a 57 meses; p <0,001) (Fig. 2).

9

\section{DISCUSIÓN}

Las características patológicas adversas como la invasión de la grasa sinusal (IGS), la invasión de la vena renal (IVR) o la invasión de grasa perinéfrica (IGP) producen tumores cT1 a pT3a independientemente del tamaño del tumor. La literatura sobre incidencia, características clínicas y resultados de los pacientes con masas renales cT1 con estadio patológico oculto T3a diagnosticadas después de la escisión quirúrgica es escasa. 1

Comunicamos una incidencia del 20,3\% (105/517) de sobreestadificación de cT1 a pT3a y el 10,6\% (27/254) sometido a NP tenía sobreestadificación. Esto fue casi similar a Ramaswamy et al., donde de 494 pacientes, 66 (13.3\%) sobreestadificaron a pT3a y 10,4\% sobreestadificaron entre pacientes con $\mathrm{NP}^{1}$. Gorin et al. informaron de una tasa de sobreestadificación de pT3a del 3,9\% entre pacientes sometidos a NP robótica que fue más baja. ${ }^{5,6}$ Tuvimos una mediana de seguimiento mayor de 88 meses para toda la cohorte que la de Ramaswamy et al. (> 48 meses) y Gorin et al. (17 meses) $)^{1,6}$ 
2 Tres $(3 / 412 ; 0.7 \%)$ pacientes que no sobreestadificaron tuvieron recurrencia en una mediana

3 de seguimiento (RIQ) de 67,5 (197,75) meses. Gorin et al. informaron que de los 41 pacientes

4 que sobreestadificaron, a pT3a después de NP, 2 tuvieron recurrencia y la estimación de 5 supervivencia libre de recurrencia fue menor para los tumores pT3a que para los tumores pT1 6 a pT2 estadificados $\left(91,8 \%\right.$ vs $99,2 \%$, respectivamente; $\mathrm{P}=0,003$ ) a los 2 años. ${ }^{6} \mathrm{Se}$ 7 diferenciaron de los nuestros en que incluían solo a aquellos pacientes con NP que 8 sobreestadificaban a pT3a con un seguimiento más corto. Solo algunas series han comparado 9 los resultados de NP y NR para masas renales con características adversas de EHP y sugieren resultados oncológicos casi similares. ${ }^{6-8}$ Se ha sugerido la validación externa de factores pronósticos para ayudar al urólogo en la toma de decisiones clínicas en el manejo de CCR..9

Todavía hay falta de claridad con respecto a la eficacia de la NP para tumores pT3a. ${ }^{6}$ Margulis et al. identificaron que la ubicación de la extensión del tumor extrarenal no afectaba la supervivencia de los pacientes con CCR pt3a y observaron que la IGS, IGP, ubicación de la extensión extrarrenal fueron insignificantes. ${ }^{5}$ El CCR pT3a se clasifica solo en función de la extensión anatómica (IGS, IGP o IVR), sin considerar el tamaño del tumor. Por lo tanto, las neoplasias pequeñas y grandes se clasifican juntas y el papel pronóstico del tamaño del tumor en la etapa CCR pT3 está siendo evaluado y se ha demostrado que el tamaño es un factor pronóstico importante.. ${ }^{4}$

En los pacientes que sobreestadificaron de cT1 a pT3a, se ha demostrado que la NP tiene un peor resultado oncológico. ${ }^{10}$ Se observó sobreestadificación de pT3a en pacientes con mayor complejidad tumoral, aumentando tamaño tumoral preoperatorio y la ubicación central cerca del sistema colector. Estos pacientes deben ser aconsejados sobre su mayor riesgo de 
1 sobreestadificación del tumor y la necesidad de NR. Se necesita un seguimiento más

2 prolongado para identificar si la NP en pT3a es suficiente para los resultados oncológicos

$3 \quad .1,11$

4

5 Méritos

6 Este es un gran estudio de un solo centro y se realizó examen histopatológico en el

7 mismo centro con una larga mediana de seguimiento. Hemos comparado la supervivencia de

8 pacientes con cT1 que sobreestadificaron a pT3a con CCR de novo pT3a.

9

\section{Limitaciones}

No tuvimos detalles sobre IGS, IGP ni IVR en todos pacientes debido a cambios en la clasificación TNM. El estadio actual pT3a no fue descrito por patólogos con anterioridad, potencialmente subestimando la incidencia de pT3a. Al ser un estudio retrospectivo, la puntuación de nefrometría no estaba disponible para todos los pacientes.

\section{CONCLUSIÓN}

La sobreestadificación patológica desde cT1 a pT3a y la necrosis en la histopatología fueron factores que llevaron a la recurrencia. Edad avanzada, tabaquismo, necrosis en la histopatología, histología de células claras y grados más altos de Fuhrman contribuyeron a la sobreestadificación patológica de los tumores cT1. El CCR de novo pT3a tuvo una supervivencia peor cuando se comparó con los pacientes con cT1 que sobreestadificaron a CCR pT3a.

Fuente de financiación: Ninguna

Conflicto de intereses: Nada que declarar 
2 BIBLIOGRAFÍA

3 1. Ramaswamy K, Kheterpal E, Pham H, Mohan S, Stifelman M, Taneja S, et al. 4 Significance of Pathologic T3a Upstaging in Clinical T1 Renal Masses Undergoing $5 \quad$ Nephrectomy. Clin Genitourin Cancer 2015;13(4):344-9.

6 2. Vilaseca A, Musquera M, Nguyen DP, Di Paola G, Romeo LR, Melnick A, et al. Changing 7 patterns in the surgical management of renal masses. Actas Urol Esp 2016;40(3):148-54.

8 3. Chevinsky M, Imnadze M, Sankin A, Winer A, Mano R, Jakubowski C, et al. 9 Pathological Stage T3a Significantly Increases Disease Recurrence across All Tumor 10 Sizes in Renal Cell Carcinoma. J Urol 2015;194(2):310-5.

4. Schiavina R, Borghesi M, Chessa F, Dababneh H, Bianchi L, Della Mora L, et al. The Prognostic Impact of Tumor Size on Cancer-Specific and Overall Survival Among Patients With Pathologic T3a Renal Cell Carcinoma. Clin Genitourin Cancer 2015;13(4):e235-41.

5. Margulis V, Tamboli P, Matin SF, Meisner M, Swanson DA, Wood CG. Location of extrarenal tumor extension does not impact survival of patients with pT3a renal cell carcinoma. J Urol 2007;178(5):1878-82.

6. Gorin MA, Ball MW, Pierorazio PM, Tanagho YS, Bhayani SB, Kaouk JH, et al. Outcomes and predictors of clinical $\mathrm{T} 1$ to pathological T3a tumor up-staging after robotic partial nephrectomy: a multi-institutional analysis. J Urol 2013;190(5):190711.

7. Jeong SH, Kim JK, Park J, Jeon HJ, Yoon MY, Jeong CW, et al. Pathological T3a Upstaging of Clinical T1 Renal Cell Carcinoma: Outcomes According to Surgical 24 Technique and Predictors of Upstaging. PLoS One 2016;11(11):e0166183. 
1 Upstaging of Clinical T1 to Pathological T3a Renal Cell Carcinoma: A Multiinstitutional

2 Analysis of Short-term Outcomes. Urology 2016;94:154-60.

3 9. Lorente D, Trilla E, Meseguer A, Planas J, Placer J, Celma A, et al. Systematic review of 4 renal carcinoma prognostic factors. Actas Urol Esp 2017;41(4):215-225.

5 10. Mouracade P, Kara O, Dagenais J, Maurice MJ, Nelson RJ, Malkoc E, et al. 6 Perioperative morbidity, oncological outcomes and predictors of pT3a upstaging for 7 patients undergoing partial nephrectomy for cT1 tumors. World J Urol 2017 Feb 14. 8 doi: 10.1007/s00345-017-2004-X.

9 11. Poon SA, Gonzalez JR, Benson MC, McKiernan JM. Invasion of renal sinus fat is not 10 an independent predictor of survival in pT3a renal cell carcinoma. BJU Int $11 \quad 2009 ; 103(12): 1622-5$. 
2 Leyendas de las figuras:

3 Fig. 1: Análisis de supervivencia de Kaplan Meier entre pacientes que sobreestadificaron de

4 cT1 a pT3a y aquellos que no lo hicieron: A - Mediana de supervivencia entre los que 5 sobreestadifican; B - Mediana de tiempo hasta recurrencia.

6

$7 \quad$ A. Log rank (Mantel Cox) 5,873

$8 \quad \mathrm{P}=0,015$

$9 \quad$ Supervivencia acumulada

10 Ninguna sobreestadificación

11 Sobreestadificación cT1 a pT3

12 Supervivencia (meses)

13

14 B. Log rank (Mantel Cox) 0,093

$15 \quad \mathrm{P}=0,761$

16 Ninguna sobreestadificación

17 Sobreestadificación cT1 a pT3

18 Tiempo hasta recurrencia (meses) 
2 Fig. 2: Análisis de supervivencia de Kaplan Meier: supervivencia entre los pacientes que 3 sobreestadificaron de cT1 a pT3a y aquellos que fueron CCR de novo pT3a.

$4 \quad$ Log rank (Mantel Cox) 31,310

$5 \quad \mathrm{P}<0,001$

6 Supervivencia acumulada

$7 \quad$ Sobreestadificación cT1 a pT3

8 De novo pT3a

$9 \quad$ Supervivencia (meses) 


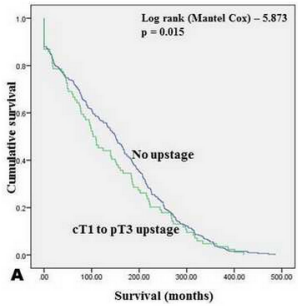

Log rank (Mantel Cox) -0.093 $p=0.761$

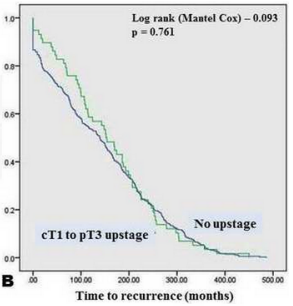


Tabla 1: Características basales y operatorias

\begin{tabular}{|c|c|c|c|c|}
\hline \multirow[b]{2}{*}{ Parámetro } & \multicolumn{3}{|c|}{$\begin{array}{c}\text { cT1 en la presentación } \\
\qquad(N=517)\end{array}$} & \multirow{2}{*}{$\begin{array}{l}\text { pT3a de novo } \\
\text { en la } \\
\text { presentación } \\
\qquad(\mathrm{N}=45)\end{array}$} \\
\hline & $\begin{array}{l}\text { Cohorte } \\
\text { completa } \\
(\mathrm{N}=517)\end{array}$ & $\begin{array}{c}\text { Sobreestadifica } \\
\text { ción de pT3a } \\
(\mathbf{N}=105)\end{array}$ & $\begin{array}{c}\text { Ninguna } \\
\text { sobreestadifica } \\
\text { ción de pT3a } \\
(\mathrm{N}=\mathbf{4 1 2})\end{array}$ & \\
\hline $\begin{array}{l}\text { Edad, Media } \pm \\
\text { DE (años) }\end{array}$ & $62,38 \pm 12,07$ & $63,98 \pm 10,67$ & $59,96 \pm 12,4$ & $69,56 \pm 11,53$ \\
\hline Hombres N (\%) & $394(76,2)$ & $79(75,2)$ & $315(76,4)$ & $35(77,8)$ \\
\hline $\begin{array}{l}\text { Índice de masa } \\
\text { corporal, Media } \\
\pm \mathrm{DE}\left(\mathrm{kg} / \mathrm{m}^{2}\right)\end{array}$ & $25,71 \pm 3,86$ & $26,08 \pm 3,74$ & $25,77 \pm 4,15$ & $25,76 \pm 4,5$ \\
\hline $\begin{array}{l}\text { Creatinina } \\
\text { sérica } \\
\text { preoperatoria, } \\
\text { Media } \pm \\
\mathrm{DE}(\mu \mathrm{mol} / \mathrm{L})\end{array}$ & $95,22 \pm 30,78$ & $93,79 \pm 28,21$ & $93,85 \pm 29,24$ & $89,31 \pm 2,1$ \\
\hline \multicolumn{5}{|l|}{ Clase ASA N (\%) } \\
\hline I & $318(61,5)$ & $48(45,7)$ & $264(64,1)$ & $21(46,7)$ \\
\hline II & $154(29,8)$ & $45(2,9)$ & $117(28,4)$ & $5(11,1)$ \\
\hline III & $45(8,7)$ & $12(11,4)$ & $31(7,5)$ & $19(42,2)$ \\
\hline $\begin{array}{l}\text { Nefrectomía } \\
\text { radical } \\
\mathrm{N}(\%)\end{array}$ & $263(50,9)$ & $55(52,3)$ & $208(50,5)$ & $45(100)$ \\
\hline $\begin{array}{l}\text { Duración de la } \\
\text { cirugía, Media } \pm \\
\text { DE (minutos) }\end{array}$ & $122,42 \pm 61,16$ & $123,35 \pm 46,5$ & $122,1 \pm 57,85$ & $124,42 \pm 40,74$ \\
\hline $\begin{array}{l}\text { Pérdida de } \\
\text { sangre } \\
\text { estimada, } \\
\text { Mediana (RIQ) } \\
(\mathrm{ml})\end{array}$ & $100(230)$ & $100(280)$ & $100(222)$ & $200(375)$ \\
\hline
\end{tabular}


Tabla 2: Factores asociados con la sobreestadificación de pT3a

\begin{tabular}{|c|c|c|c|}
\hline Parámetro & $\begin{array}{l}\text { Sobreestadificaci } \\
\text { ón de pT3a } \\
(\mathrm{N}=105)\end{array}$ & $\begin{array}{l}\text { Ninguna } \\
\text { sobreestadificació } \\
\text { n de pT3a } \\
(\mathrm{N}=412)\end{array}$ & Valor de $p$ \\
\hline $\begin{array}{l}\text { Edad, Media } \pm \text { DE } \\
\text { (años) }\end{array}$ & $63,98 \pm 10,67$ & $59,96 \pm 12,4$ & 0,006 \\
\hline $\begin{array}{l}\text { Tamaño T preoperatorio, } \\
\text { Media } \pm \text { DE }(\mathrm{cm})\end{array}$ & $44,19 \pm 15,42$ & $37,77 \pm 14,04$ & $<0,001$ \\
\hline $\begin{array}{l}\text { Tamaño T postoperatorio, } \\
\text { Media } \pm \text { DE }(\mathrm{cm})\end{array}$ & $53,18 \pm 19,03$ & $36,08 \pm 15,32$ & $<0,001$ \\
\hline Tabaquismo, $\mathrm{N}(\%)$ & $41(39)$ & $105(25,5)$ & 0,044 \\
\hline Necrosis, N (\%) & $15(14,3)$ & $27(6,6)$ & 0,010 \\
\hline $\begin{array}{l}\text { CCR células claras, } \mathrm{N} \\
(\%)\end{array}$ & $89(84,8)$ & $302(73,3)$ & 0,015 \\
\hline $\begin{array}{l}\text { Primera recurrencia, } \mathrm{N} \\
(\%)\end{array}$ & $9(8,6)$ & $3(0,7)$ & $<0,001$ \\
\hline
\end{tabular}


Tabla 3: Clasificación de Fuhrman y clase ASA y sobreestadificación patológica

\begin{tabular}{|c|c|c|c|}
\hline Grado de Fuhrman & $\begin{array}{l}\text { Sobreestadificación } \\
\text { de pT3a } \\
(\mathrm{N}=105) \\
\mathrm{N}(\%)\end{array}$ & $\begin{array}{l}\text { Ninguna } \\
\text { sobreestadificación } \\
\text { de pT3a } \\
(\mathrm{N}=412) \\
\mathrm{N}(\%)\end{array}$ & Valor de $p$ \\
\hline 1 & $4(3,8)$ & $22(5,3)$ & \multirow{4}{*}{$<0,001$} \\
\hline 2 & $44(41,9)$ & $272(66)$ & \\
\hline 3 & $46(43,8)$ & $108(26,3)$ & \\
\hline 4 & $11(10,5)$ & $10(2,4)$ & \\
\hline \multicolumn{4}{|l|}{ Clase ASA } \\
\hline 1 & $35(1,9)$ & $189(1,2)$ & \multirow{4}{*}{0,003} \\
\hline 2 & $13(1,9)$ & $81(4,1)$ & \\
\hline 3 & $45(41,9)$ & $109(66)$ & \\
\hline 4 & $12(43,8)$ & $33(26,3)$ & \\
\hline
\end{tabular}

